\title{
Role of Laparoscopy in Management of Ectopic Pregnancy
}

\author{
Hasina Banu ${ }^{1}$, Ju Wen $\mathrm{Hui}^{2}$, Liu Hua ${ }^{3}$ \\ Received: February 15, 2015 Accepted: August 16, 2015 \\ doi: http://dx.doi.org/10.3329/jemc.v5i3.24750
}

\begin{abstract}
Ectopic pregnancy means implantation of fertilized ovum outside the endometrial lining of the uterus. It remains the leading cause of early pregnancy-related death. Delay in diagnosis and treatment puts the life of women at risk. Laparoscopic surgery is increasingly becoming the preferred approach for ectopic pregnancy management. Laparoscopic treatment in ectopic pregnancy raises question of safety and feasibility when compared to laparotomy. In this review article our objective is to summarize the role of laparoscopy in management of ectopic pregnancy in comparison to laparotomy. For this, a literature search was done by using Google and PubMed. The selected articles were analyzed on laparoscopic treatment outcomes such as surgery success rate, operating time, intraoperative and postoperative complications, hospital stay, future fertility, postoperative recurrent ectopic pregnancy, cost-effectiveness in comparison to laparotomy. After analyzing all selected articles, it can be concluded that the laparoscopic management of ectopic pregnancy is safe, effective, and economical in comparision to laparotomy. So, for the patients' benefit, laparoscopy should be considered as the gold standard method in management of ectopic pregnancy and is worthy to be popularized in clinical practice.
\end{abstract}

Key words: Ectopic pregnancy; Laparoscopy; Laparotomy

J Enam Med Col 2015; 5(3): 170-174

\section{Introduction}

An ectopic pregnancy is defined as implantation of a fertilized ovum outside the endometrial lining of the uterus. ${ }^{1}$ The most common site of ectopic pregnancy is tubal pregnancy which includes $98 \%$ of all ectopic pregnancies. ${ }^{2}$ The ectopic pregnancy remains the leading cause of early pregnancy related death in first trimester. ${ }^{3}$ The incidence of ectopic pregnancy has increased substantially over the past decades with increasing trends of risk factors and improvement in the diagnostic modalities. Several developments in treatment of ectopic pregnancy have led to remarkable success in the mother's life. Further developments recently have resulted in a shift in focus from saving the mothers' life to additionally saving the women's fertility. ${ }^{4}$ Traditionally, ectopic pregnancy was treated by laparotomy. John Burd, a surgeon in New York, reported the first successful surgical intervention to treat ectopic pregnancy in $1759 . .^{5}$ Currently laparoscopy is gaining significant popularity in management of ectopic pregnancy. ${ }^{5-7}$ Laparoscopic surgery was first reported by Bhuhout et al in $1980 .{ }^{8}$ Laparoscopic surgery has advantage of shorter operative time, less blood loss, fewer analgesic requirements, shorter hospital stay and more cost effective than laparotomy. There were fewer

1. Resident, Department of Gynecology and Obstetrics, Jingzhou Central Hospital, Affiliated to Yangtze University Medical College, Jingzhou 434023, Hubei Province, P.R. China

2. Department of Gynecology and Obstetrics, Jingzhou Central Hospital, Affiliated to Yangtze University Medical College, Jingzhou 434023, Hubei Province, P.R. China

3. Department of Gynecology and Obstetrics, Jingzhou Central Hospital, Affiliated to Yangtze University Medical College, Jingzhou 434023, Hubei Province, P.R. China

Correspondence Hasina Banu, Email: drbanuh@gmail.com 
repeated ectopic pregnancies in women treated laparoscopically although this is not significant. ${ }^{1}$ Laparoscopic salphingectomy and laparoscopic salphingostomy are usually being performed for ectopic pregnancy management.

\section{Procedures}

All the procedures are done under general anesthesia in semilithotomy position. Laparoscopy is performed by using 4 ports after establishing pneumoperitoneum with carbon dioxide insufflators $12-14 \mathrm{~mm} \mathrm{Hg}$. Through an intraumbilical incision, trocars are introduced followed by introduction of $10 \mathrm{~mm}$ laparoscope. After confirmation of the diagnosis $5 \mathrm{~mm}$ incision is given in the left and the right lower quadrant under direct visualization and transillumination to avoid the epigastric vessels. Another $10 \mathrm{~mm}$ incision is given at subumblicus in hypochondrium of abdomen. Salphingectomy is done stepwise desiccation of the mesosalpinx with bipolar forcep and cutting along the mesosalpinx proximal to the tube using scissors. For salphingostomy linear incision is given on the prominent and distended antimesenteric border of tube with scissors after coagulating with bipolar forcep. The products of conceptions are removed with tooth grasper. The incision can be left open to allow heal by secondary intension or defects are repaired. The surgical specimens are removed by $10 \mathrm{~mm}$ subumbilical trocar sleev. And at the end peritoneal washings are done with normal saline.

\section{Results}

There are lots of researches done to determine which one is better treatment for ectopic pregnancy laparoscopy or laparotomy.

1. Bramsted et al study at the University of Vermont. This study was retrospective case control involving 101 cases of ectopic pregnancy conducted between 1982-1987. This study compared the difference between laparoscopy and laparotomy in their outcomes. Twenty five patients were treated by laparoscopy and 76 by laparotomy. Patients' selections were done on the basis of hemodynamic status (stable and unstable). Hemodynamically stable patients were managed by laparoscopy and unstable patients were managed by laparotomy. The author found that the patients treated by laparoscopic surgery required less operating time, fewer analgesic requirement, shorter hospital stay and early recovery than laparotomy treated group. ${ }^{10}$

2. Vermesh et al study. This prospective study was designed to compare prospectively the parameters of morbidity, cost, length of hospital stay, and fertility outcome after linear salphingostomy by laparoscopy vs laparotomy. Patients selection were made on the basis of stable vital signs, hematocrit greater than $30 \%$, age over 18 years, and desire for future fertility. All patients underwent diagnostic laparoscopy. Sixty patients with unruptured ectopic pregnancy of $5 \mathrm{~cm}$ or less were randomized equally to laparoscopic treatments or laparotomy. The two groups had similar beta-HCG levels. The estimated blood loss was significantly less in laparoscopy group. Two patients in the laparoscopy group required laparotomy postoperatively. Histosalphingogram demonstrated similar patency of the treated fallopian tubes. Eighty four per cent of laparoscopy and $89 \%$ of the laparotomy had patent tubes. Moreover, the pregnancy rates were $56 \%$ in laparoscopy and $58 \%$ in laparotomy. Six months following surgery, $56 \%$ of the patients treated by laparoscopy and $58 \%$ of the patients treated with laparotomy conceived spontaneously. ${ }^{11}$

3. Yao \& Tulandi showed that the subsequent intrauterine pregnancy was $70 \%$ in laparoscopytreated patients and 55\% in laparotomy-treated patients. $^{12}$

4. There was one follow-up study about adhesions after laparoscopic surgery and after laparotomy. There were 105 patients with tubal pregnancy. They were stratified with regards to age, risk factors and randomized to surgery by laparoscopy or laparotomy. Seventy three patients with strong desire of pregnancy underwent a second look laparoscopy. The adhesion status at the ipsilateral and contralateral side at the primary surgery was compared. The author found that the patients who had undergone laparoscopic treatment for the previous ectopic pregnancy had less adhesion than the laparotomy treated group patients. But the tubal patency were same in both groups. ${ }^{13}$

5. Another study, conducted by Xiang in China, on 142 patients compared the result of laparoscopic surgery and laparotomy in management of ectopic 
pregnancy. Seventy patients were treated by laparotomy and 72 patients by laparoscopy. They found that the patients who were treated laparoscopically has less operative time and less postoperative hospital stay in comparison to laparotomy group patients and laparoscopic treatment for ectopic pregnancy was well accepted by both the surgeons and the patients. ${ }^{14}$

6. Similarly in a study of 105 patients in Prince of Wales Hospital in Hong Kong, Yuen reported in 1997, the result of review of laparoscopy and laparotomy in the treatment of ectopic pregnancy. Sixty one patients were treated by laparoscopy and 44 patients by laparotomy. Age, parity, gestational age, frequency of prior ectopic pregnancy and previous laparotomy were similar for the two groups. The laparoscopy group had $45.9 \%$ hemoperitoneum and the laparotomy group had $75 \%$ hemoperitoneum. The duration of postoperative hospital stay was 2-9 days for laparoscopy group and 51 days for the laparotomy group. They concluded that the advantage of laparoscopy is in combining diagnostic and therapeutic procedures in a single procedure, and is a better approach than laparotomy in the surgical treatment of ectopic pregnancy. ${ }^{15}$

7. There was one more study conducted by Murphy et al at the San Diego school of medicine. It was a prospective study. They compared laparoscopy and laparotomy in the management of hemodynamically stable patients. A total of 63 patients were included in the study of which 26 patients underwent laparoscopy and 37 patients underwent laparotomy. The result reported that there were short hospital stay, less blood loss, fewer postoperative analgesic requirement, early recovery and less total hospital cost in comparison to the laparotomy group patients. ${ }^{16}$

8. EL-Tabbakh reported the result of a trial in Kuwait from March 1999-October 2001, involving 207 patients to compare laparoscopy vs laparotomy for surgical treatment of ectopic pregnancy. One hundred eighty four patients were treated by laparoscopy and 23 by laparotomy. The diagnosis of 207 patients was based on clinical symptoms, history, physical examination, positive serum beta HCG, TVS, and ectopics were confirmed at laparoscopy. Following surgery the patients were followed with serial quantitative beta HCG on day $4^{\text {th }}$ and day $7^{\text {th }}$, then weekly until the levels were less than $20 \mathrm{IU} / \mathrm{L}$. The patients treated with laparoscopy had an overall success rate $98.9 \%$. Moreover, the patients treated by laparoscopy had significantly less blood loss, blood transfusion was required only in $13 \%$ patients in the laparoscopic treated group compared to $23 \%$ in the laparotomy group patients. The author concluded that laparoscopy treatment is superior to laparotomy with less blood loss. Therefore, patients required less transfusion and less analgesics and a shorter postoperative hospital stay. ${ }^{17}$

9. Clasen et al conducted a study in Belgium involving 293 cases where they did only laparoscopic management and the results favored a laparoscopic approach. Unfortunately laparotomy had to be performed in eight cases due to intense hemorrhage and advanced gestational age. Among these eight cases three were primary and five were converted from laparoscopy. A total of 14 cases remained with residual diseases and were treated either by methotrexate or a second surgical procedure. This study evaluated that the overall rate of spontaneous conceptions was $77.3 \%$ and there was a $10.6 \%$ recurrent rate of ectopic pregnancy. The author concluded that laparoscopy approach should remain the gold standard in treating ectopic pregnancy. ${ }^{18}$

10. The department of Gynecology and Obstetrics, E. Wolfson Medical Center, Israel, designed a parallel study on hemodynamically unstable patients. One hundred and one patients with ectopic pregnancy underwent laparoscopic surgery. Of these 18 patients had sustained intra-abdominal bleeding and clinical signs and symptoms of hemodynamic unstablity. These patients underwent laparoscopic salphingectomy and only one required conversion to laparotomy. There were no any major intraoperative or postoperative complications, and all the patients made a full and uneventful recovery. The study was concluded with the statements that improved anesthesia and cardiovascular monitoring, combined with advanced laparoscopic surgical skills and experience, justifies operative laparoscopy for the surgical treatment of ectopic pregnancy even in women who are hemodynamically unstable. ${ }^{19}$ 
11. With the introduction of laparoscopic surgery to the Gynecology and Obstetrics department at South Cleveland Hospital, the department decided to review their management of ectopic pregnancy. A retrospective analysis of 210 cases of ectopic pregnancy were managed over a period of 5 years, operative findings and surgical data were analyzed. One hundred seventy seven patients were managed successfully by laparoscopy with no major intraoperative or postoperative complications. Thirty three patients were managed by laparotomy for various reasons. The estimated blood loss, need for transfusion and the length of hospital stay following laparoscopic treatment were significantly less than those in laparotomy group. This study demonstrated that laparoscopic management of ectopic pregnancy is the most beneficial procedure with maximum safety. ${ }^{20}$

\section{Discussion}

There are lots of studies on the management of ectopic pregnancy in the literature ranging from case reports to randomized trials, from conservative management to radical surgery. Now-a-days laparoscopic surgery is accepted as a gold standard for the surgical management of ectopic pregnancy unless it is absolutely contraindicated. Laparoscopic surgeries have lots of benefits for the patients in comparison to laparotomy.

Clasen et a ${ }^{18}$ did only laparoscopic management in 194 cases of ectopic pregnancy with $97.4 \%$ success rate. Some other series of studies also confirm the success rate of operative laparoscopic surgery in ectopic pregnancy between $87-97 \% .^{16,20-22}$ Some authors had performed operative laparoscopy even in hemodynamically unstable patients with good success rate. ${ }^{19}$

Many authors conducted a randomized, prospective, retrospective clinical trial on duration in laparoscopy and laparotomy and found that laparoscopic surgery required less time than laparotomy. ${ }^{10,13,14}$ One study found that the laparoscopic surgery took 73 minutes vs. 88 minutes in laparotomy group. ${ }^{23}$

Chatwani et $\mathrm{al}^{9}$ conducted a nonrandomized study and found significant decrease in blood transfusion rate in laparoscopic group. Similar results were found in several other studies. ${ }^{11,16,17,20}$ Some articles also showed that postoperatively the requirement for analgesic was significantly less in laparoscopic group patients. ${ }^{10,16}$ And also found less adhesions after laparoscopic surgery. ${ }^{4}$

There are many studies which reported a much shorter hospital stay following laparoscopic surgery and therefore, it proves to be cost-effective. . $^{10,11,14-17,20,21}$

Laparoscopic surgery had let to a better convalescence in form of shorter hospital stay, faster recovery, and decreased need for analgesic, cosmetically good scar and less psychological trauma to the patients.

From literature we know that laparoscopic surgery is a more cost-effective approach. An average savings of approximately US\$1,200-1,500 for each patient can be obtained, because it significantly reduces the length of hospital stay. ${ }^{16,24}$

The concern for future fertility poses a debate as there is a certain amount of tubal damage, but both methods of surgery have has comparable pregnancy rate. ${ }^{11}$ Overall conception rate is $77.3 \% .^{18}$

\section{Conclusion}

Critical overview of literatures demonstrates that the laparoscopy has greater role in managing ectopic pregnancy, is safe and effective challenging alternative to laparotomy. Therefore, laparoscopy should be opted whenever possible for management of ectopic pregnancy. In future the conventional laparotomy will be replaced by laparoscopic approach for almost all cases of ectopic pregnancy.

\section{References}

1. Ectopic pregnancy. In: Hoffman BL, Schorge JO, Schaffer JI, Halvorson LM, Bradshaw KD, Cunningham FG (eds). Williams gynecology. $2^{\text {nd }}$ edn. New York: McGraw Hill, 2012: 198-212.

2. Ectopic gestation. In: Padubidri VG, Daftany SN (eds). Howkins \& Bourne Shaw's textbook of gynaecology. $15^{\text {th }}$ edn. New Delhi: Elsevier, 2011: 265-288.

3. Dutta DC. Hemorhage in early pregnancy. In: Textbook of obstetrics. $6^{\text {th }}$ edn. Calcutta: New Cetral Book Agency (P) Ltd., 2008: 159-202.

4. Tenore JL. Ectopic pregnancy. Am Fam Physician 2000; 61(4): 1080-1088.

5. Sepilian V. Ectopic pregnancy Differential diagnoses. Available at: http://emedicine.medscape.com/article/ 2041923-differential. Accessed June 2015. 
6. Odejinmi FO, Rizzuto MI, Macrae RE, Thakur V. Changing trends in the laparoscopic management of ectopic pregnancy in London District General Hospital: 7 years experience. J Obstet Gynaecol 2008; 28: 614-617.

7. Hsu MI, Tang CH, Hsu PY, Huang YT, Long CY, Huang KH et al. Primary and repeated surgeries for ectopic pregnancies and distribution by patient age, surgeon age and hospital levels: an 11 years nationwide population based descriptive study in Taiwan. J Minim Invasive Gynecol 2012; 19: 598-605.

8. Bruhat MA, Manhes H, Mage G, Poully JL. Treatment of ectopic pregnancy by means of laparoscopy. Fertil Steril 1980; 33: 411-414.

9. Chatwani A, Yazigi R, Amin-Hanjanis. Operative laparoscopy in the management of tubal ectopic pregnancy. $\mathrm{J}$ Laparoscopic Surg 1992; 2(6): 319-324.

10. Brumsted J, Kessler E, Gibon E, Nakajima S, Riddick DH, Gibson M. A comparison of laparoscopy and laparotomy for the treatment of ectopic pregnancy. Obstet Gynecol 1988; 71: 889-902.

11. Vermesh M, Silva PD, Rosen GF, Stein AL, Fossum GT, Sauer MV. Management of unruptured ectopic gestation by linear salpingectomy: a prospective randomized clinical trial of laparoscopy versus laparotomy. Obstet Gynecol 1989; 73: 400-404.

12. Yao M, Tulandi T.Current status of surgical and non-surgical management of ectopic pregnancy. Fertil Steril 1997; 67: 421-433.

13. Lundorff P, Hahlin M, Kallfelt B, Thorburn J, Lindblom B. Adhesion formation after laparoscopic surgery in tubal pregnancy: a randomized trial versus laparotomy. Fertil Steril 1991; 55: 911-915.

14. Xiang XD, Tane YQ. Treatment of ectopic pregnancy. Singapore Med J 1999; 40(2): 88-90.
15. Yuen PM, Rogers MS, Chang A. A review of laparoscopy and laparotomy in the management of tubal pregnancy. Hong-Kong Med J 1997; 3(2): 153-157.

16. Murphy AA, Nager CW, Wujek JJ, Kettel MM, Torp VA, Chin HG. Operative laparoscopy versus laparotomy for the management of ectopic pregnancy: a prospective trial. Fertil Steril 1992; 57: 1180-1185.

17. El-Tabbakh MN, Elsays MS. Tubal Ectopic Pregnancy; Laparoscopy vs Laparotomy. Available at: http://www.obgyn net/ivf/tubal-ectopic-pregnancy-laparoscopy-vs-laparotomy. Accessed January 2015.

18. Clasen K, Camus M, Tournaye H, Devroey P. Ectopic pregnancy: let's cut! strict laparoscopic approach to 194 consecutive cases and review of literature and alternatives. Human Reproduction 1997; 12(3): 596-601.

19. Sagiv R, Debby A, Sandano O, Malinger G, Glezerman M, Golan A. Laparoscopic surgery for extrauterine pregnancy in hemo-dynamically unstable patients. J AM Assoc Gynecol laproscopic 2001; 8(4): 529-532.

20. Mohamed H, Maiti S, Phillips G.. Laproscopic management of ectopic pregnancy: a 5 year experience. J Obstet Gynaecol 2002; 22(4): 411-414.

21. Zouves C, Urman B, Gomel V. Laparoscopic surgical treatment of tubal pregnancy. A sage, effective alternative to laprotomy. J Reprod Med 1992; 37(3): 205-209.

22. Chapron C, Querleu D, Crepin G. Laproscopic treatment of ectopic pregnancies; a one hundred case study. Eur J Obstet Gynecol Reprod Biol 1991; 41(3): 187-190.

23. Steiner RA, Ioannidis K, Weigh E, Fehr M, Haller V. Minimally invasive surgery in the treatment of extrauterine pregnancy. Schweiz Rundsch Med Preax 1993; 82: 1117-1121.

24. Duggal BS, Tarneja P, Sharma RK, Rath SK, Wadhwa RD. Laparoscopic management of ectopic pregnancies. MJAFI 2004; 60: 220-223. 